\title{
The fracture behaviors of carbon nanotube and nanoscroll reinforced silicon matrix composites
}

\author{
Hao Chen, Shaohua Chen * \\ LNM, Institute of Mechanics, Chinese Academy of Sciences, Beijing 100190, China
}

\section{A R T I C L E I N F O}

Article history:

Received 25 July 2013

Accepted 3 October 2013

Available online 10 October 2013

\begin{abstract}
A B S T R A C T
Fracture properties of both carbon nanotube (CNT) and carbon nanoscroll (CNS) reinforced silicon (Si) matrix composites under tension are investigated by molecular dynamics simulations. It is found that either a single-wall CNT or a multi-wall one (MWCNT) will be pulled out if the length of the CNT is short, while brittle fracture of CNT will happen for a relatively long one. It is interesting to find that the "sword-in-sheath" fracture mode observed experimentally in a long MWCNT reinforced alumina matrix composite is verified well by our simulations. Furthermore, comparing to a CNT reinforced Si matrix composite, fracture toughness of a CNS reinforced one can be significantly enhanced by both the length and the layer of the CNS. Crack in CNS propagates along its circumference and moves inward layer by layer so that large parts of the fracture energy are dissipated. The results provide a direct understanding of the fracture strength observed experimentally and an insight for improving the fracture toughness of some novel composites.
\end{abstract}

(c) 2013 Elsevier Ltd. All rights reserved.

\section{Introduction}

As a novel material, CNT reinforced composites should possess many potential applications due to the outstanding mechanical, chemical and electrical properties of CNT [110]. However, results of the composite's strength and toughness are disappointing $[10,11]$, presumably owing to the inhomogeneous dispersion of CNTs in matrix and weak interface between two phases. Plenty of efforts have been devoted to understanding the CNT/ceramic interface both experimentally and computationally [10,12-14]. For example, experiments have found that MWCNTs would fail with a 'swordin-sheath' fracture mode rather than pull-out in a MWCNT reinforced aluminum matrix composite [10]. Numerical simulations have discussed the effective Young's modulus and interface strength of a CNT reinforced Si matrix composite and demonstrated that both could be highly improved by an increasing bond strength between CNT fibers and Si matrix [12]. However, what is the mechanical mechanism of the

\footnotetext{
* Corresponding author: Fax: +86 1082543977.

E-mail address: chenshaohua72@hotmail.com (S. Chen).
} 0008-6223/\$ - see front matter (c) 2013 Elsevier Ltd. All rights reserved. http://dx.doi.org/10.1016/j.carbon.2013.10.004 'sword-in-sheath' failure mode? Does the length of reinforcing CNTs influence the failure mode?

In addition, similar to CNTs, CNS as a new material based on graphene sheets, has a helical structure and has attracted great research interests recently $[15,16]$. Recently, a simple method with the assistance of microwave sparks in liquid nitrogen has been proved to be valid in producing high-quality CNSs from graphene [16]. Unlike MWCNT possessing a weak interaction between the layers, CNS has a continuous curly structure. Is there a different effect on the fracture property of a silicon matrix composite if we utilize CNSs as a novel reinforcement instead of CNTs?

\section{Simulation methods}

In order to answer the above questions, the fracture properties of both CNTs and CNSs reinforced silicon matrix composites are investigated with molecular dynamics (MD) simulations. The C-C bond length for both CNTs and CNSs is $0.142 \mathrm{~nm}$. Si is chosen as a representative diamond matrix 


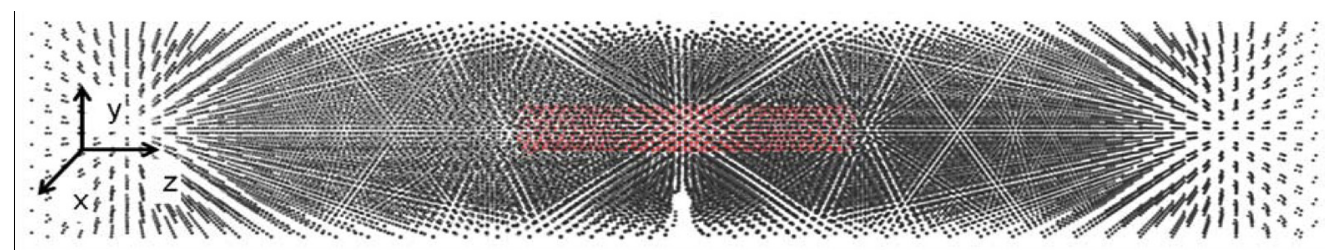

(a)

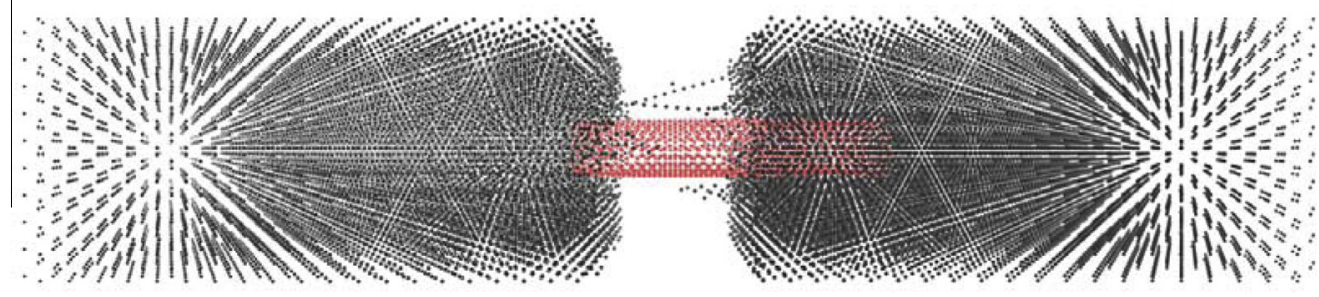

(b)

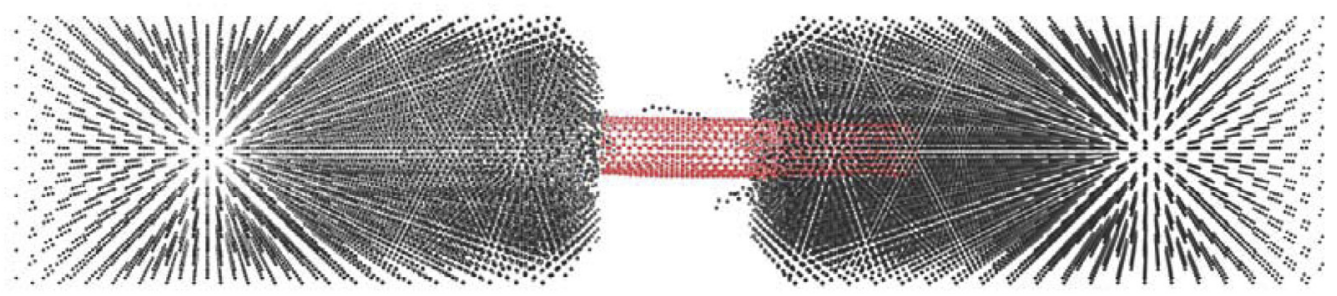

(c)

Fig. 1 - Snapshots of the failure process of a SWCNT reinforced Si matrix composite under external tension, where the length of the SWCNT is $l=7.3 \mathrm{~nm}$. (a) With strain of 0.21 . Here, the strain is defined as the external displacement divided by the initially axial length of the unit cell model. (b) With strain of $\mathbf{0 . 2 2}$. (c) With strain of 0.23 . (A color version of this figure can be viewed online.)

and the [100] direction of Si matrix is parallel to the CNTs or CNSs axis with laterally periodic boundary conditions. The method of inserting a CNT or CNS into Si matrix is very similar to that in $[12,14]$, with a $0.19 \mathrm{~nm}$ distance between the $\mathrm{C}$ atoms on the outer layer of CNTs or CNSs and the $\mathrm{Si}$ atoms.

A unit cell of a CNT reinforced Si matrix composite is shown in Fig. 1(a), where the size of the simulation model is $5.431 \times 5.431 \times 21.723 \mathrm{~nm}^{3}$ and a crack of length $1.0 \mathrm{~nm}$ is pre-set at the edge of Si matrix for the fracture study. All simulations are performed using the large-scale atomic molecular massively parallel simulator (LAMMPS) [17]. The Erhart/Albe-Tersoff potential is adopted to describe interactions between $\mathrm{Si}-\mathrm{Si}$ atoms, C-C atoms as well as C-Si atoms, which has been confirmed in accordance with the firstprinciple results [18]. The equations of motion are solved using a velocity-Verlet algorithm with a time step of $1.0 \mathrm{fs}$. Before loading, each sample is relaxed at $10 \mathrm{~K}$ for $5.0 \mathrm{ps}$ with a Nose-Hoover thermostat to achieve equilibrium. Then, a tensile displacement $(0.005 \mathrm{~nm} / \mathrm{ps})$ in the axial direction is applied on one end of the matrix and the other end keeps fixed as shown in Fig. 1(a).

\section{Results and discussions}

The effect of single-wall CNT's (SWCNT) length on the failure behavior of a Si matrix composite is studied at first, where the SWCNT is armchair type with chirality of (10, 10). A series of simulations are carried out and we find that the failure process of a SWCNT reinforced Si matrix composite is significantly influenced by the length of SWCNT. Figs. $1(\mathrm{a}-\mathrm{c})$ show the snapshots of failure process of the SWCNT reinforced Si matrix composite with the SWCNT's length $l=7.3 \mathrm{~nm}$. Fig. 1 (a) presents the state before the matrix crack propagates. With further tension, the crack in $\mathrm{Si}$ matrix propagates until the cross-section of the whole matrix fails. Then, the interface between SWCNT and Si will sustain the external loading. Due to the short interface length, sliding of SWCNT will happen as shown in Fig. 1(b), where the SWCNT bridges the two parts of matrix. With the external loading increasing, SWCNT is finally pulled out from the Si matrix as shown in Fig. 1(c). In addition, the fracture surface roughness and Si single atomic chains bridging the two fracture surfaces are clearly seen in Fig. 1, which should be due to the artifact of atomic potential. The unphysical phenomena can also be found in the fracture surfaces below.

However, if the length of SWCNT is long enough, the interface could sustain the external loading. As a result, the interface between SWCNT and Si matrix keeps perfect without sliding. In this case, equilibrium equation of the tensile stress $\sigma_{\mathrm{f}}$ in SWCNT and the interface shear stress $\tau_{\mathrm{e}}$ can be described by the shear-lag model $[19,20]$, 
$S \frac{\mathrm{d} \sigma_{\mathrm{f}}}{\mathrm{dz}}=p \tau_{\mathrm{e}}$

where the diameter and thickness of the SWCNT are $D=1.34 \mathrm{~nm}$ and $h=0.0617 \mathrm{~nm}$, respectively. Then, the area of SWCNT's cross section is $S=\pi D h$ and the perimeter is $p=\pi D$ [21]. In addition, from the simulation results, we observe an obvious detachment of CNT at both ends with small contact areas. So the tensile stress at both ends of SWCNT is negligible and we assume the tensile stress at both ends of SWCNT vanishes, i.e., $\sigma_{\mathrm{f}}=0$. Integrating Eq. (1) yields the maximum tensile stress at the middle of SWCNT,

$\sigma_{\mathrm{fm}}=p \tau_{\mathrm{e}} \mathrm{l} / 2 \mathrm{~S}$

When the maximum tensile stress achieves its tensile strength $\sigma_{\mathrm{s}}$, the SWCNT would fail due to fracture.

Fig. 2 gives some snapshots for the failure process of a long SWCNT reinforced Si matrix composite under tension. The length of SWCNT in Fig. 2 is $l=18.2 \mathrm{~nm}$. Fig. 2(a) is the state of a SWCNT reinforced Si matrix composite, before the Si matrix crack propagates. After further external tension, the crack in Si matrix propagates, which leads to the fracture of Si matrix as shown in Fig. 2(b). Further increasing the external loading, SWCNT fails due to brittle fracture while the interface between the SWCNT and Si keeps perfect owing to its long length as shown in Fig. 2(c). Notice that from equation (2), we can obtain the interface shear strength: $\tau_{\mathrm{e}}=542 \mathrm{MPa}$ when the tensile strength of CNT is $80 \mathrm{GPa}$.
The interface between CNT and silicon is also investigated. A Si-C covalent bond is identified when the distance between the two atoms is less than $0.195 \mathrm{~nm}$ [22]. The geometrical pattern of covalent Si-C bonds is shown in Fig. 3. The bond density is identified to be $3.8287 \mathrm{~nm}^{-2}$. When the distance of the two atoms is out of the range, they interact with each other through van der waals forces [22].

The failure behaviors of double-wall CNTs (DWCNTs) of (5, $5)$ and $(10,10)$ reinforced Si matrix composites are further simulated. Similar results to SWCNT cases in Fig. 1 can be found if the length of DWCNT is relatively short, for example $l=7.3 \mathrm{~nm}$. However, a 'sword-in-sheath' failure behavior, which was observed experimentally [10] is also found in our MD simulations as shown in Fig. 4 when the length of DWCNT is relatively long, for example $l=18.2 \mathrm{~nm}$. Fig. 4 (a) gives the state of a DWCNT reinforced Si matrix composite before the matrix crack propagates. With further tension, the Si matrix first fails by fracture as shown in Fig. 4(b), at which time the interface sustains the external loading and transfers the loading to the outer layer of DWCNT. When the axial stress in DWCNT exceeds the theoretical strength, the outer layer of DWCNT fails by fracture. However, due to the weak interface between the outer layer and the inner one of DWCNT, intersliding which looks like a 'sword-in-sheath' happens as shown in Fig. 4(c) [23].

Fig. 5 shows the stress-strain curves of both SWCNT and DWCNT reinforced Si matrix composites. The Young's modulus is found to increase with the length of CNT, which is

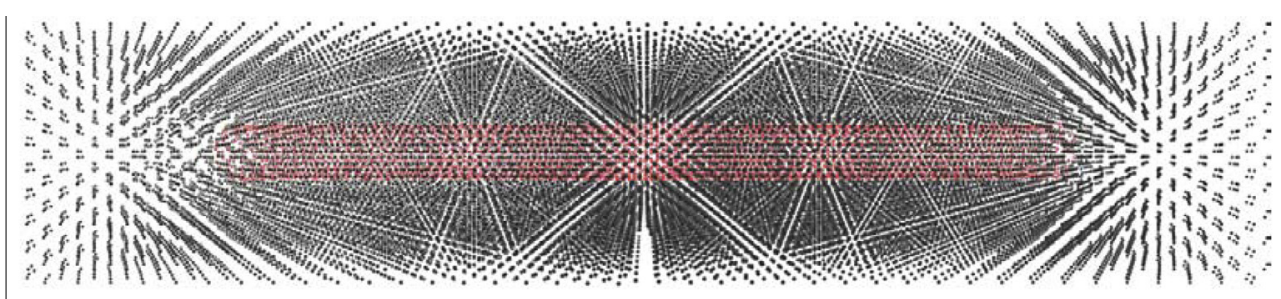

(a)

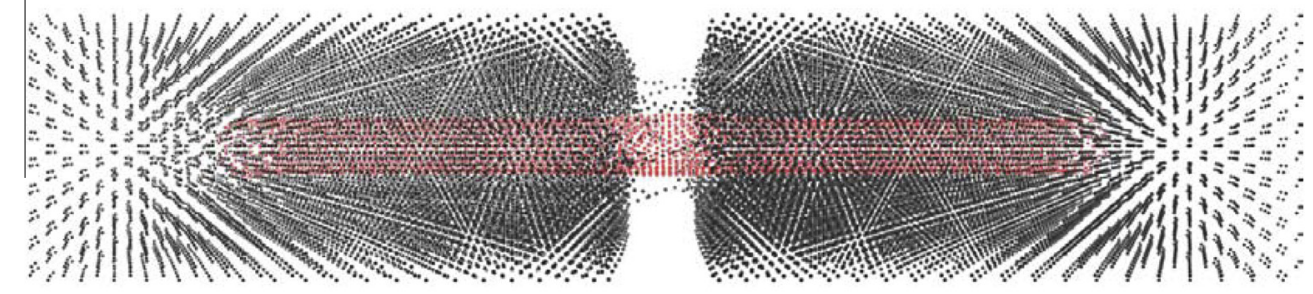

(b)

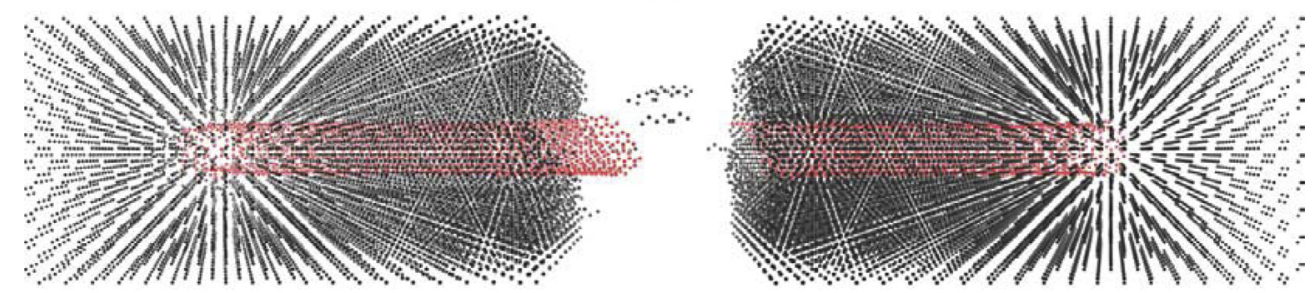

(c)

Fig. 2 - Snapshots of the failure process of a single-wall CNT reinforced Si matrix composite under external tension, where the length of the SWCNT is $l=18.2 \mathrm{~nm}$. (a) With strain of 0.2 . (b) With strain of 0.21 . (c) With strain of 0.22 . (A color version of this figure can be viewed online.) 


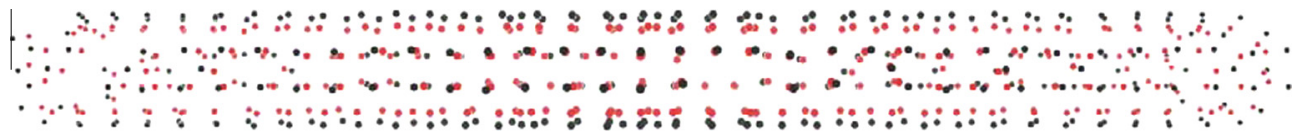

Fig. 3 - Geometrical patterns of covalent Si-C bonds formed at the CNT-Si interface, where the length of CNT is $\mathrm{l}=18.2 \mathrm{~nm}$. Black dots denote $\mathrm{Si}$ atoms and the red ones denote $\mathrm{C}$ atoms, respectively. (A color version of this figure can be viewed online.)

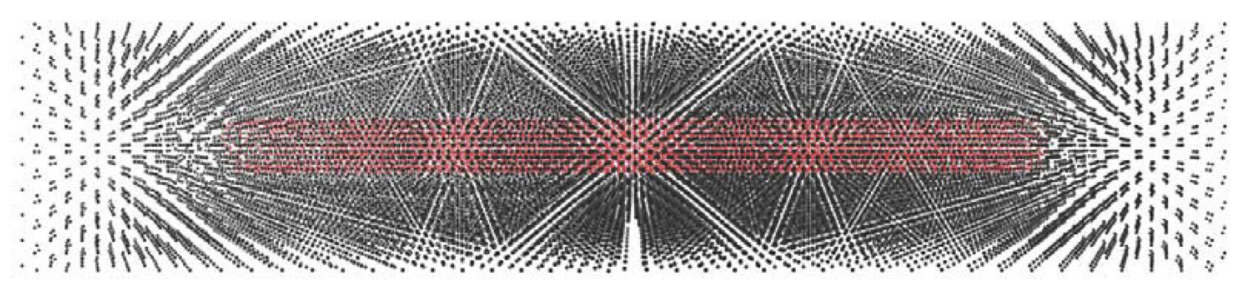

(a)

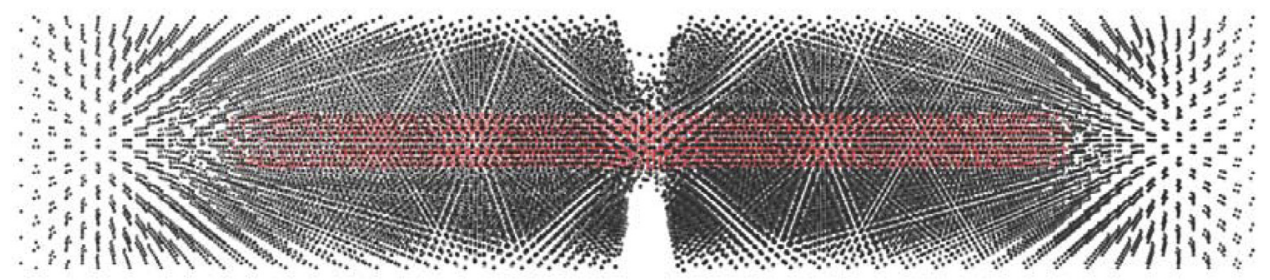

(b)

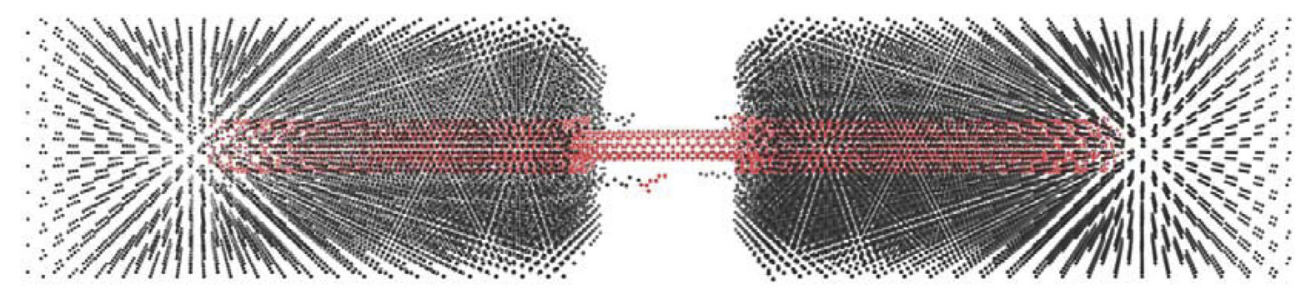

(c)

Fig. 4 - Snapshots of the failure process of a DWCNT reinforced Si matrix composite under external tension, where the length of the double-wall CNT is $l=18.2 \mathrm{~nm}$. (a) With strain of 0.2 . (b) With strain of 0.21 . (c) With strain of 0.22 . (A color version of this figure can be viewed online.)

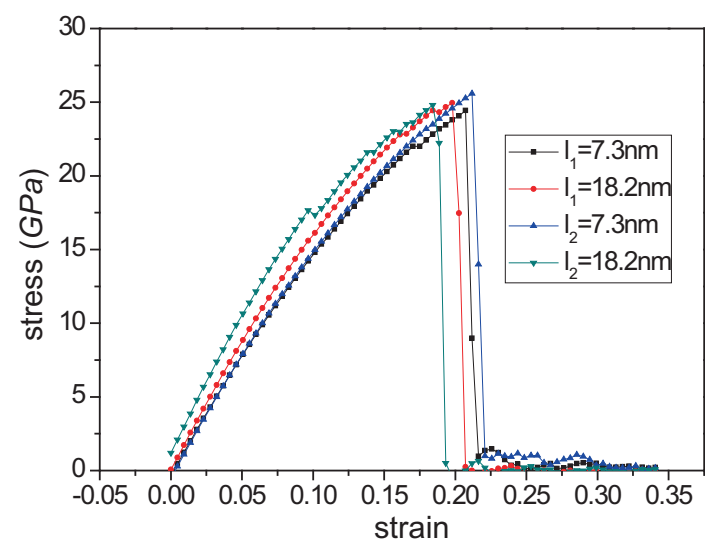

Fig. 5 - The stress-strain curves of Si composites reinforced by a single-wall CNT with a length $l_{1}$ and that reinforced by a double-wall one with a length $l_{2}$. (A color version of this figure can be viewed online.)

consistent well with the theoretical predictions [24] and the previous simulations $[25,26]$. The pop-in effects occurring in some of the stress vs. strain curves as shown in Fig. 5 are mainly due to two facts: one is the breaking of some of the interface covalent bonds and the other is the separation of $\mathrm{C}$ atoms from Si ones at both ends of the CNT. The stress decreases abruptly when the matrix starts to fracture, which denotes the quick propagation of the crack and totally brittle fail of the composite. The strength of the composite keeps almost the same either with the reinforcing SWCNT or DWCNT because the interlayer slide of MWCNTs does not show any obvious influence. The results demonstrate that either a MWCNT or a SWCNT cannot enhance the toughness of silicon matrix, providing a reasonable explanation for the experimental results [10].

How can we enhance the toughness of such a brittle matrix? As a recently new material, CNSs may show some insight, which have been successfully fabricated through microwave spark assistance in liquid nitrogen [16]. In the present paper, CNSs of armchair type with interlayer distance $t=0.34 \mathrm{~nm}$ and outer radius $R=1.86 \mathrm{~nm}$ are used to replace CNTs in the $\mathrm{Si}$ matrix. Size of the unit cell is $10.862 \times 10.862 \times 21.723 \mathrm{~nm}^{3}$ with a pre-set crack of length $3.73 \mathrm{~nm}$ in the Si matrix. In order to achieve CNSs of different 
layers, we change the inner radius. Snapshots of the failure process of a Si matrix composite reinforced by a CNS of four layers with the length $l=18.2 \mathrm{~nm}$ are shown in Fig. 6. Initially, the Si matrix fails by crack propagation as shown in Fig. 6(a). Then the Si matrix fails totally with the increasing external extension and the interface between CNS and Si matrix sustains the external loading and transfers it to CNS. As a result, a crack forms in the out-layer of CNS along the zigzag direction and propagates along the circumference of CNS as shown in Fig. 6(b). With the external extension increasing, crack in the CNS propagates layer by layer until the CNS fails as shown in Fig. 6(c). Wrinkle is produced in the CNS under the shear stress, which is also found in free-standing graphene [27]. An interesting phenomenon is that CNS demonstrates a tremendously bridging effect even for a $45 \%$ strain in Fig. 6(c), unlike the brittle fracture in a CNT reinforced Si matrix composite. For a double-layer CNS, once the Si matrix fails, crack produced in the CNS would propagate and spread over the whole cross section of CNS very soon, leading to a small facture toughness of the composite as shown in Fig. 7.

Comparing to the CNT reinforced Si matrix composite, the CNS reinforced one possesses much larger fracture toughness. As shown in Fig. 8, while the strength of Si reinforced by different CNSs does not change too much, the fracture toughness can be improved by the number of layers of CNS due to the bridging effect, where the length is fixed for both a DWCNT and CNSs, i.e., $l=18.2 \mathrm{~nm}$. The fracture toughness of a material is related to the crack propagation path. Crack

(a)

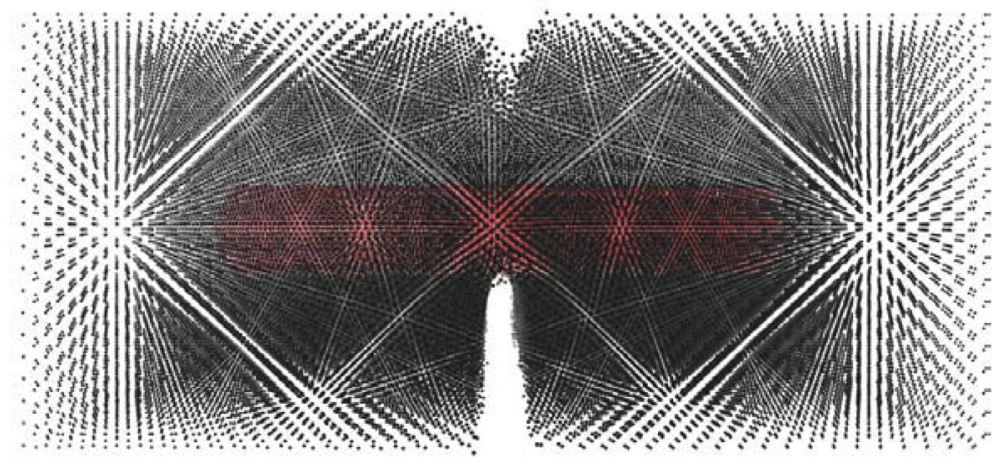

(b)

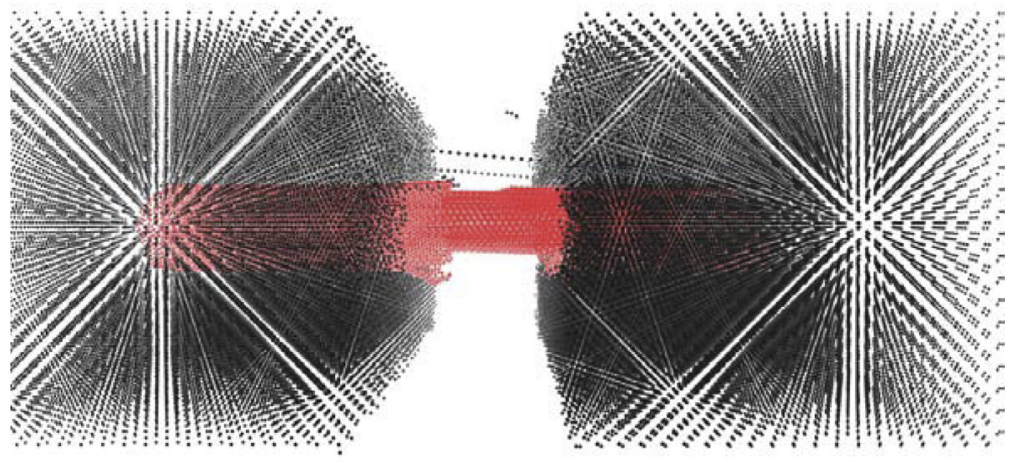

(c)

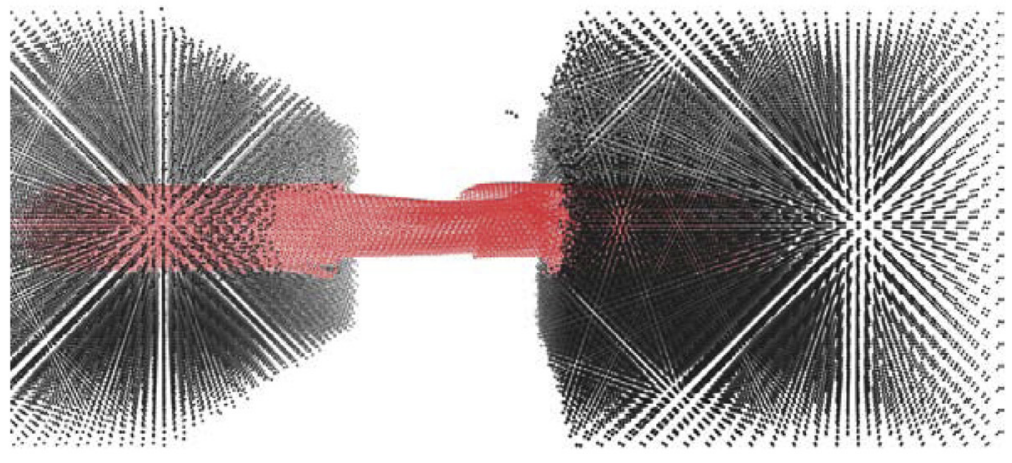

(d)

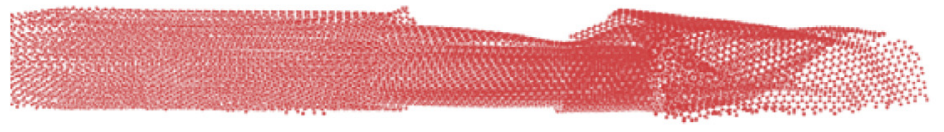

Fig. 6 - Snapshots of the failure process of a four-layer CNS reinforced Si matrix composite under external tension, where the length of the CNS is $l=18.2 \mathrm{~nm}$. (a) With strain of 0.117 . (b) With strain of 0.237 . (c) With strain of 0.43 . (d) The configuration of the deformed CNS in the composite in (c). (A color version of this figure can be viewed online.) 


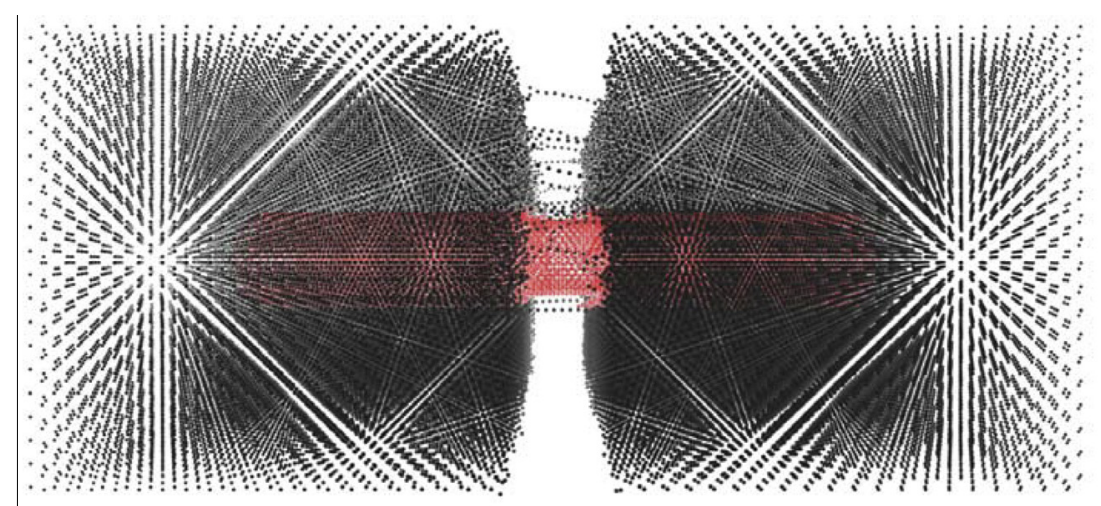

(a)
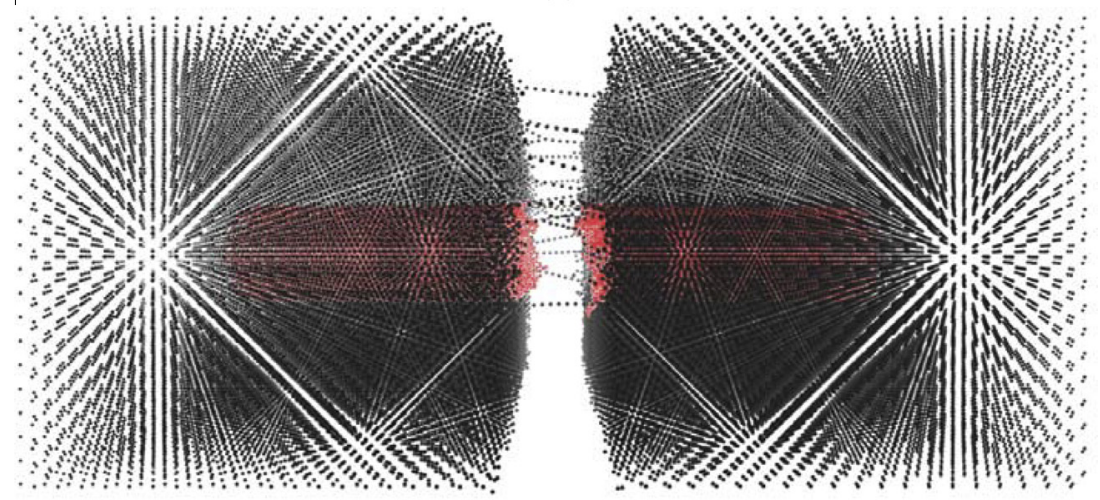

(b)

Fig. 7 - Snapshots of the failure process of a double-layer CNS reinforced Si matrix composite under external tension, where the length of the CNS is $l=18.2 \mathrm{~nm}$. (a) With strain of 0.127 . (b) With strain of 0.137 . (A color version of this figure can be viewed online.)

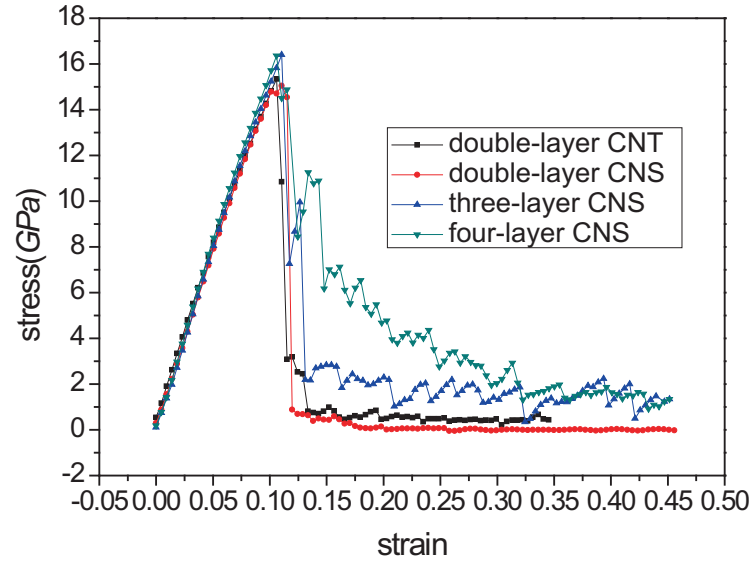

Fig. 8 - Stress-strain curves of Si composites reinforced by different layered CNSs but with the same length $l=18.2 \mathrm{~nm}$. The one of a DWCNT reinforced Si matrix composite is given for comparison. (A color version of this figure can be viewed online.)

forming in the CNS propagates along the circumference, which would dissipate large parts of the strain energy. The more the number of CNS layers, the larger the fracture toughness of Si reinforced by CNSs is. Our simulation results dis- close that the dramatically increasing fracture toughness in CNS reinforced $\mathrm{Si}$ matrix composite is mainly due to the bridging effect resulted from the multi-layer CNS [28]. Furthermore, it can be inferred that not only the fracture strength but also the fracture toughness of a material with a relatively low strength reinforced by long and multi-layer CNSs could be enhanced significantly. This idea should be useful for the development of new composites.

However, the fracture toughness of a CNS reinforced composite can be enhanced only if the interface between the CNS and Si matrix does not slide. It means that the length of the interface should not be too short to sustain the external loading. Simulations are also carried out for a Si matrix composite reinforced by CNSs with a length of $l=7.3 \mathrm{~nm}$ but with different layers. Similar to the failure form of a composite with a short SWCNT or DWCNT, the short CNSs will be pulled out as shown in Fig. 9, leading to a nearly brittle fracture of the composite. The stress-strain relationships for four-layered CNSs but with different lengths are shown in Fig. 10. It is found that the longer the CNSs, the better the toughening effect is.

\section{Conclusions}

In conclusion, the fracture behaviors of both CNT and CNS reinforced Si matrix composites are investigated. It is found 


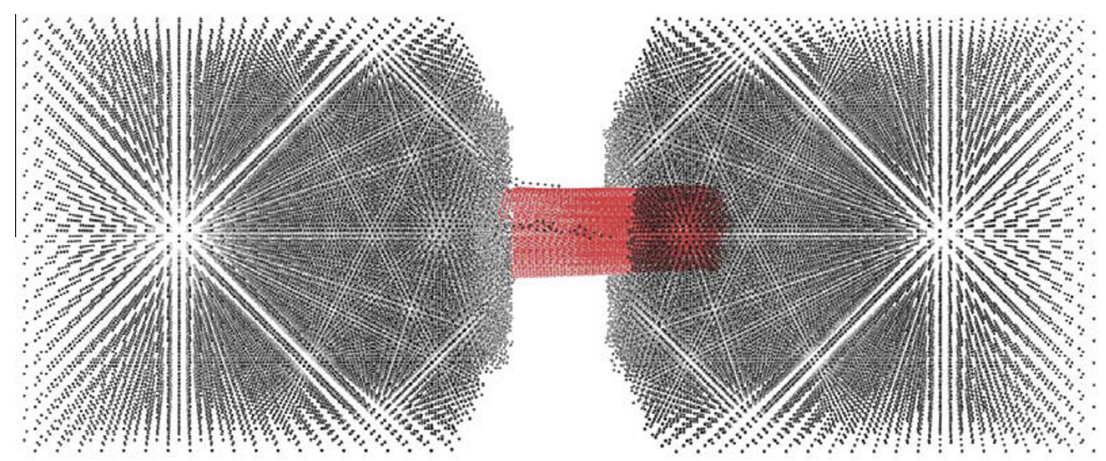

Fig. 9 - Snapshot of a four-layer CNS with length $l=7.3 \mathrm{~nm}$ pulled out from the Si matrix composite. (A color version of this figure can be viewed online.)

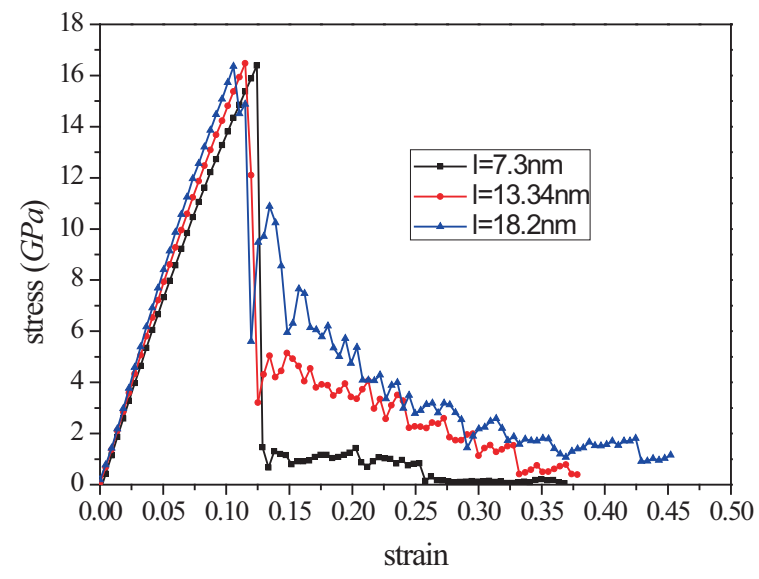

Fig. 10 - Stress-strain curves of Si composites reinforced by four-layer CNSs with different lengths. (A color version of this figure can be viewed online.)

that either a short SWCNT or MWCNT reinforced Si matrix composite fails by brittle fracture due to the brittle failure of $\mathrm{Si}$ matrix accompanying the simultaneous pull-out of CNT. In addition for a long SWCNT or MWCNT reinforced Si matrix composite, it also has a brittle fracture owing to the brittle fracture of Si matrix with the simultaneous fracture of SWCNT or the 'sword-in-sheath' failure mode of MWCNT. In order to achieve a better fracture toughness, a new reinforcing phase is proposed in this paper. Simulations results demonstrate that the fracture toughness of a CNS reinforced Si matrix composite can be significantly enhanced by long and multi-layers CNSs. Our results not only provide reasonable explanations on the experiment observation of a special 'sword-in-sheath' failure mode and the fact that the toughness of CNT reinforced diamond composites is disappointing, but also propose a novel reinforcing material CNS which has a new toughening mechanism for carbon composites.

\section{Acknowledgements}

Support from the National Science Foundation of China (Grant No. 10972220, 11125211, 11021262) and the 973 Nanoproject (2012CB937500) is gratefully acknowledged.
R E F E R E N C E S

[1] Ebbesen TW, Lezec HJ, Hiura H, Bennett JW, Ghaemi HF, Thio T. Electrical conductivity of individual carbon nanotubes. Nature 1996;382:54-6.

[2] Treacy MMJ, Ebbesen TW, Gibson JM. Exceptionally high Young's modulus observed for individual carbon nanotubes. Nature 1996;381:678-80.

[3] Yakobson BI, Brabec CJ, Bernholc J. Nanomechanics of carbon tubes: instabilities beyond linear response. Phys Rev Lett 1996;76:2511-4.

[4] Yakobson BI, Campbell MP, Brabec CJ, Bernholc J. High strain rate fracture and $\mathrm{C}$-chain unraveling in carbon nanotubes. Comput Mater Sci 1997;8:341-8.

[5] Ajayan PM, Tour JM. Materials science - nanotube composites. Nature 2007;447:1066-8.

[6] Baughman RH, Zakhidov AA, de Heer WA. Carbon nanotubes - the route toward applications. Science 2002;297:787-92.

[7] Balani K, Agarwal A. Damping behavior of carbon nanotube reinforced aluminum oxide coatings by nanomechanical dynamic modulus mapping. J Appl Phys 2008;104:063517.

[8] Cha SI, Kim KT, Lee KH, Mo CB, Hong SH. Strengthening and toughening of carbon nanotube reinforced alumina nanocomposite fabricated by molecular level mixing process. Scr Mater 2005;53:793-7.

[9] Ma RZ, Wu J, Wei BQ, Liang J, Wu DH. Processing and properties of carbon nanotubes-nano-SiC ceramic. J Mater Sci 1998;33:5243-6.

[10] Yamamoto G, Shirasu K, Hashida T, Takagi T, Suk JW, An J, et al. Nanotube fracture during the failure of carbon nanotube/alumina composites. Carbon 2011;49:3709-16.

[11] Sheldon BW, Curtin WA. Nanoceramic composites: tough to test. Nat Mater 2004;3:505-6.

[12] Kim BH, Lee KR, Chung YC, Lee JG. Effects of interfacial bonding in the Si-carbon nanotube nanocomposite: a molecular dynamics approach. J Appl Phys 2012;112:044312.

[13] Li L, Niu JB, Xia ZH, Yang YQ, Liang JY. Nanotube/matrix interfacial friction and sliding in composites with an amorphous carbon matrix. Scripta Mater 2011;65:1014-7.

[14] Pavia F, Curtin WA. Interfacial sliding in carbon nanotube/ diamond matrix composites. Acta Mater 2011;59:6700-9.

[15] Xie X, Ju L, Feng XF, Sun YH, Zhou RF, Liu K, et al. Controlled fabrication of high-quality carbon nanoscrolls from monolayer graphene. Nano Lett 2009;9:2565-70.

[16] Zheng J, Liu HT, Wu B, Guo YL, Wu T, Yu G, et al. Production of high-quality carbon nanoscrolls with microwave spark assistance in liquid nitrogen. Adv Mater 2011;23:2460-3.

[17] Plimpton S. Fast parallel algorithms for short-range molecular-dynamics. J Comput Phys 1995;117:1-19. 
[18] Erhart P, Albe K. Analytical potential for atomistic simulations of silicon, carbon, and silicon carbide. Phys Rev B 2005;71:035211.

[19] Nairn JA. On the use of shear-lag methods for analysis of stress transfer in unidirectional composites. Mech Mater 1997;26:63-80.

[20] Gao XL, Li K. A shear-lag model for carbon nanotubereinforced polymer composites. Int J Solids Struct 2005;42:1649-67.

[21] Huang Y, Wu J, Hwang KC. Thickness of graphene and singlewall carbon nanotubes. Phys Rev B 2006;74:245413.

[22] Sorkin V, Zhang YW. Rotation-dependent epitaxial relations between graphene and the Si-terminated SiC substrate. Phys Rev B 2010;82:085434.

[23] Liu P, Zhang YW, Lu C, Lam KY. Tensile and bending properties of double-walled carbon nanotubes. J Phys D: Appl Phys 2004;37:2358-63.
[24] Shokrieh MM, Rafiee R. Investigation of nanotube length effect on the reinforcement efficiency in carbon nanotube based composites. Compos Struct 2010;92:2415-20.

[25] Zhu R, Pan E, Roy AK. Molecular dynamics study of the stress-strain behavior of carbon-nanotube reinforced Epon 862 composites. Mater Sci Eng A 2007;447:51-7.

[26] Zhu R, Pan E, Chung PW, Cai X, Liew KM, Buldum A. Atomistic calculation of elastic moduli in strained silicon. Semicond Sci Technol 2006;21:906-11.

[27] Min K, Aluru NR. Mechanical properties of graphene under shear deformation. Appl Phys Lett 2011;98:013113.

[28] Pei QX, Zhang YW, Shenoy VB. A molecular dynamics study of the mechanical properties of hydrogen functionalized graphene. Carbon 2010;48:898-904. 